\title{
PINCH1 knockout aggravates myocardial infarction in mice via mediating the $\mathrm{NF}-\kappa \mathrm{B}$ signaling pathway
}

\author{
XUEJUN WANG ${ }^{1 *}$, JINWEN SU $^{2 *}$, ZHIKANG LIN ${ }^{1}$, KANGYONG LIU $^{3}$ and YU ZHUANG ${ }^{4}$ \\ ${ }^{1}$ Department of Cardiology, Shanghai University of Medicine and Health Sciences Affiliated Zhoupu Hospital, \\ Shanghai 201318; ${ }^{2}$ Department of Cardiology, Shanghai Ninth People's Hospital Affiliated to \\ Shanghai JiaoTong University School of Medicine, Shanghai 200011; ${ }^{3}$ Department of Neurology, \\ Shanghai University of Medicine and Health Sciences Affiliated Zhoupu Hospital, Shanghai 201318; \\ ${ }^{4}$ Department of Cardiovascular Surgery, Shanghai General Hospital, Shanghai Jiao Tong \\ University School of Medicine, Shanghai 200080, P.R. China
}

Received May 9, 2020; Accepted October 12, 2021

DOI: $10.3892 /$ etm.2021.10984

\begin{abstract}
Myocardial infarction (MI), the leading cause of death among patients with cardiovascular diseases, is characterized by acute cardiac muscle injury due to severe impairment of the coronary blood supply, which may lead to cardiogenic shock and cardiac arrest. Particularly interesting new cysteine histidine rich 1 (PINCH1) protein, a key component of the integrin signaling pathway, interacts with several proteins and serves a vital role in numerous cellular processes, including cytoskeleton remodeling, cell proliferation and cell migration. To investigate the role of PINCH1 in heart injury in the present study, PINCH1 was knocked out in the myocardial tissue of mice (age, 18 weeks) to induce MI. In addition, cell viability, migration and apoptosis, as well as the expression levels of $\mathrm{NF}-\kappa \mathrm{B}$-associated proteins were determined in murine HL1 cardiomyocytes with a conditional PINCH1 shRNA using Cell Counting Kit-8, Transwell, flow cytometry and western blot assays, respectively. Furthermore, the cardiac expansion and myocardial fibrosis in PINCH1 knockout mice was investigated in vivo by performing morphological and histological examinations. Additionally, the murine ventricular myocardial ultrastructure was evaluated using an electron microscope, and the cardiomyocyte apoptotic rate and expression levels of $\mathrm{NF}-\kappa \mathrm{B}-$ related proteins were determined using TUNEL and western blot assays, respectively. The results showed that the
\end{abstract}

Correspondence to: Dr Yu Zhuang, Department of Cardiovascular Surgery, Shanghai General Hospital, Shanghai Jiao Tong University School of Medicine, 100 Haining Road, Shanghai 200080, P.R. China

E-mail: yuzhuang0301@126.com

${ }^{*}$ Contributed equally

Key words: particularly interesting new cysteine histidine rich 1, myocardial infarction, NF- $\mathrm{B}$ signaling pathway, cardiomyopathy, HL1 cell line apoptotic rate in the in vivo PINCH1 knockdown group was significantly increased. In addition, the protein expression levels of $\mathrm{NF}-\kappa \mathrm{B}$ signaling pathway-related proteins, including $\mathrm{NF}-\kappa \mathrm{B}$, myeloid differentiation factor 88 , TNF- $\alpha$ and caspase-3, were significantly increased in the in vivo PINCH1 knockdown group compared with the wild-type group, but the protein expression of MMP2 and MMP9 were the opposite. Overall, the in vitro and in vivo results revealed that PINCH1 knockout in mice significantly aggravated MI via the $\mathrm{NF}-\kappa \mathrm{B}$ signaling pathway.

\section{Introduction}

Myocardial infarction (MI), a cardiovascular disease, is the primary cause of morbidity and mortality worldwide. MI is characterized by a reduction in oxygen supply caused by blocked coronary arteries, resulting in diastolic and systolic pressure dysfunction. The disease is accompanied by several mechanical complications, and the primary clinical treatment strategies aim to restore blood supply to maintain normal oxygen levels (1-7). Particularly interesting new cysteine histidine rich (PINCH) protein, a type of focal adhesion proteins in mammals, is a LIM-domain-only adapter protein consisting of two isomers, PINCH1 and PINCH2, which share high homology. PINCH serves an important role in maintaining the structure and function of the heart, and is involved in several biological processes, including cell migration and survival. PINCH1 is widely expressed in most tissues and organs, particularly in the myocardium (8-10). A study demonstrated that conditional PINCH knockout $(\mathrm{KO})$ in mice led to severely defective cardiac development. Furthermore, PINCH1 deletion resulted in impaired cell mobility and survival, whereas the functional complex formed by PINCH1, thymosin $\beta 4$ ( $\mathrm{t} \beta 4$ ) and integrin-linked kinase (ILK), promoted cardiomyocyte migration and survival following cardiac injury (1-8). The aforementioned studies indicated that PINCH1 may serve a key role in cardiac function. However, the potential molecular mechanism underlying the effects of PINCH1 in the myocardium is not completely understood.

$\mathrm{NF}-\kappa \mathrm{B}$ belongs to a family of transcription factors involved in the regulation of cell differentiation, proliferation 
and apoptosis. As an inflammatory factor, NF- $\kappa \mathrm{B}$ serves an important role in the immune system $(11,12)$. Studies $(13-15)$ have shown that the $\mathrm{NF}-\kappa \mathrm{B}$ signaling pathway is activated following $\mathrm{MI}$ and promotes ventricular remodeling in rats (16). The activity of $\mathrm{NF}-\kappa \mathrm{B}$ can be blocked by $\mathrm{t} \beta 4$, a cell penetrating peptide with a variety of biological functions, thus improving cardiac function $(1,17,18)$. However, the association between $\mathrm{PINCH} 1$ and the NF- $\mathrm{KB}$ signaling pathway in MI in mice is not completely understood. Therefore, the present study adopted multiple search methods, including western blotting, reverse transcription-quantitative (RT-q) PCR and flow cytometry, to find the correlation between PINCH1 and NF-KB pathway.

\section{Materials and methods}

Cell culture. HL1 cardiomyocytes (American Type Culture Collection) were cultured in high-glucose DMEM (Thermo Fisher Scientific, Inc.) supplemented with 10\% FBS (Thermo Fisher Scientific, Inc.) and $1 \%$ penicillin and streptomycin at $37^{\circ} \mathrm{C}$ with $95 \%$ air and $5 \% \mathrm{CO}_{2}$.

Plasmid construction and cell transfection. PINCH1 gene knockdown in cardiomyocytes was performed by transfecting HL1 cells with PINCH1 shRNA as previously described (19).

The full-length open reading frame of PINCH1 was cloned into the pCDH-CMV-MCS-EF1A-GFP-T2A-Puro vector (System Biosciences, LLC) to generate the corresponding overexpression (OE) vector, pCDH-PINCH1-OE (System Biosciences, LLC). The shRNA sequence specifically directed against PINCH1 was designed and constructed with the following targeting interference sequence (Sangon Biotech Co., Ltd.): (Forward PINCH1 shRNA 5'-3') GATCCGGAGACG CAACCAGGTCTTGCTTCAAGAGAGCAAGACCTGGTT GCGTCTCCTTTTTTG; (Reverse PINCH1 shRNA 5'-3') AAT TCAAAAAAGGAGACGCAACCAGGTCTTGCTCTCTTG AAGCAAGACCTGGTTGCGTCTCCG and were cloned into PLVX-shRNA1 (Thermo Fisher Scientific, Inc.). The negative control (NC) shRNA scrambled sequence was (Sangon Biotech Co., Ltd.): (Forward PINCH1 NC 5'-3'): GATCCGGAGAAA TTCCGGTAGCGCTTGCTTCAAGAGAGCTTGGAAATT CCTGCGTCTCCTTTTTTG; (Reverse PINCH1 NC 5'-3'): AATTCAAAAAAGTTCCGGTTAAGCTAGTCTTGCTCT CTTATTGCGCTATTCGGGTTGCGT CTCCG and were cloned into PLVX-shRNA1 (Thermo Fisher Scientific, Inc.).

At $60-70 \%$ confluence, HL1 cells were transfected with plasmids using Lipofectamine ${ }^{\circledR} 2000$ (Invitrogen; Thermo Fisher Scientific, Inc.) according to the manufacturer's protocol. Briefly, cells were treated with $4 \mu \mathrm{g}$ PCDH and $4 \mu \mathrm{g}$ PLVX-shRNA1 when the $\mathrm{HL} 1$ reached $60-70 \%$ confluency at $37^{\circ} \mathrm{C}, 5 \% \mathrm{CO}_{2}$. shRNA NC, shRNA PINCH1, pCDH-NC OE and pCDH-PINCH1 OE vectors were mixed with $500 \mathrm{ml}$ of Opti-MEM and $3.5 \mathrm{ml}$ of Lipofectamine ${ }^{\circledR}$ RNAiMAX (Invitrogen; Thermo Fisher Scientific, Inc.), respectively, and then added to a $3.5 \mathrm{~cm}$ dish. At $48 \mathrm{~h}$ post-transfection, transfection efficiencies were assessed via flow cytometric analysis, RT-qPCR and western blotting. The following successfully transfected cells were generated: shRNA NC, shRNA PINCH1, pCDH-NC OE and pCDH-PINCH1 OE.

Cell viability assay. To determine HL1 cell viability, a Cell Counting Kit 8 (CCK-8) assay was used according to the manufacturer's instructions (Beyotime Institute of Biotechnology). Briefly, cells were seeded ( $1 \times 10^{5}$ cells/well) into 96-well plates for $24 \mathrm{~h}$. Subsequently, $10 \mu \mathrm{l} \mathrm{CCK}-8$ solution was added into each well at the $72 \mathrm{~h}$. After culture for $4 \mathrm{~h}$, the optical density of each well was measured at a wavelength of $450 \mathrm{~nm}$ using an enzyme mark analyzer. Cell viability was calculated according to the following formula: Cell viability $(\%)=[($ mean OD value of PINCH1 KO group/HL1 group)/(mean OD value of HL1 group)] x100.

Transwell migration assay. The migration abilities of PINCH1 KO HL1 and control HL1 cells were assessed by performing Transwell assays. Briefly, cells were plated ( $1 \times 10^{5}$ cells/chamber, ) into the upper chamber and were cultured in $\alpha$-MEM, 10\% FBS, $10 \mathrm{U} / 1$ penicillin and $10 \mu \mathrm{g} / \mathrm{l}$ streptomycin, whereas $\alpha$-MEM medium supplemented with $20 \%$ FBS was plated in the lower chamber as a chemoattractant. Following incubation for $48 \mathrm{~h}$ at $37^{\circ} \mathrm{C}$, migratory cells were fixed with $4 \%$ paraformaldehyde at $25^{\circ} \mathrm{C}$ for $4 \mathrm{~h}$ and then stained with $0.5 \%$ crystal violet at $25^{\circ} \mathrm{C}$ for $15 \mathrm{~min}$. The number of migratory cells was quantified under a microscope (CX43; Olympus Corporation) on five randomly selected fields.

Flow cytometry. Cell apoptosis was evaluated by flow cytometry using an Annexin V-FITC/PI apoptosis kit (BD Biosciences) according to the manufacturer's protocol. After cell harvesting, $100 \mu \mathrm{l}$ binding buffer was added to prepare a cell suspension ( $1 \times 10^{6}$ cells $\left./ \mathrm{ml}\right)$. Subsequently, cells were stained with $5 \mu 1$ FITC-Annexin V and $5 \mu \mathrm{l}$ PI at room temperature for $15 \mathrm{~min}$ in the dark. Cells were then resuspended in $400 \mu \mathrm{l}$ binding buffer and analyzed by flow cytometry (FACSCalibur; Bio-Rad Laboratories, Inc.). Cells in early and late apoptosis was quantified using FlowJo 7.6.1 software (FlowJo LLC).

Western blotting. Total proteins were extracted from HL1 cells or mouse myocardial tissues using RIPA lysis buffer (Beyotime Institute of Biotechnology) supplemented with protease and phosphatase inhibitors (Beyotime Institute of Biotechnology). Protein concentrations were determined using the BCA Protein Detection kit (Beyotime Institute of Biotechnology). Equal amounts of protein $(30 \mu \mathrm{g})$ were separated via $10 \%$ SDS-PAGE and transferred onto PVDF membranes. Following blocking with $5 \%$ skim milk powder for $1 \mathrm{~h}$ at $25^{\circ} \mathrm{C}$, the membranes were incubated at $4^{\circ} \mathrm{C}$ overnight with primary antibodies targeted against: GAPDH (ab9485,1:5,000; Abcam), NF-кB (ab32360,1:1,000; Abcam), MYD88 (ab219413,1:1,000; Abcam), MMP-2 (ab92536,1:1,000; Abcam), MMP-9 (ab76003,1:1,000; Abcam), TNF- $\alpha$ (ab183218,1:1,000; Abcam),caspase-3 (ab184787,1:1,000; Abcam) and PINCH1 (ab108609,1:1,000; Abcam). Subsequently, the membranes were incubated with corresponding secondary antibodies [Goat Anti-Rabbit IgG H\&L (HRP); ab6721, 1:2,000; Abcam] for $1 \mathrm{~h}$ at room temperature. Finally, the ECL detection system was used to detect the immunoreactive protein signals and analyzed with Image J (v1.8.0.112; National Institutes of Health). GAPDH was used as the loading control.

TUNEL staining. Cell apoptosis in myocardial tissues was detected by performing TUNEL staining assays. Briefly, mouse myocardium tissue was fixed with $4 \%$ paraformaldehyde at 
$25^{\circ} \mathrm{C}$ for $4 \mathrm{~h}$. Subsequently, TUNEL staining solution (Roche Diagnostics) was added at $25^{\circ} \mathrm{C}$ for $1 \mathrm{~h}$ in a dark humid chamber. DAPI was added dropwise and incubated at $25^{\circ} \mathrm{C}$ for 5 min. TUNEL ${ }^{+}$cells were counted with mounting medium (Thermo Fisher Scientific, Inc.) and the cells were observed under microscope (DM4B; Leica Microsystems GmbH) for three fields.

MI mouse model. PINCH1 Knockout mice were generated at the transgenic core facility at University of California at San Diego. In the present study, all experimental procedures involving experimental animals were carried out in compliance with the local animal welfare laws/policies, and the approval from the local animal ethics committee of Shanghai General Hospital, Affiliated to Shanghai Jiao Tong University School of Medicine was obtained (approval number pkj2017-Y38). Adult C57BL/6 mice (weighing $20 \pm 2$ g, aged $7 \pm 1$ weeks) were ordered from Shanghai SLAC Laboratory Animal Co., Ltd. Mice were maintained in standard cages under a specific pathogen free (SPF) environment, with 12-h light/dark cycle and free access to food and water for $\sim 1$ week to acclimatize. Mice were divided into four groups: Wild-type, wild-type-MI, PINCH1 knockout group and PINCH1 knockout MI group, six mice in each group. In the wild-type (normal group) only the chests were opened and no heart surgery performed. Mice were anesthetized with $2 \%$ isoflurane (maintenance, $1.5 \%$ isoflurane) through a face mask and placed in a supine position in the center of the operating table. A midline neck incision was performed to expose the heart. The left anterior descending branch of the mouse heart was ligated under a stereoscopic microscope. After the mice are raised for four weeks, they are weighed with an electronic scales. An electrocardiograph was carried out by ultrasound on each mouse.

Histological analysis. Heart tissues were fixed with $4 \%$ polyformaldehyde for $24 \mathrm{~h}$ at $25^{\circ} \mathrm{C}$, followed by paraffin embedding. Subsequently, the paraffin-embedded sections $(3 \mu \mathrm{m})$ were stained with H\&E following dewaxing in two baths of xylene for $10 \mathrm{~min}$ each at $25^{\circ} \mathrm{C}$ and rehydration in a graded alcohol series for $5 \mathrm{~min}$ at $25^{\circ} \mathrm{C}$ before rinsing with running water for $5 \mathrm{~min}$, three times at $25^{\circ} \mathrm{C}$. Hematoxylin staining was for $5 \mathrm{~min}$, followed by $5 \%$ acetic acid differentiation for $1 \mathrm{~min}$ and washing in water at $25^{\circ} \mathrm{C}$.

Sections were also stained using Masson's trichrome; following the operations as aforesaid. The stained sections were both examined under the Leica DM5000 light microscope (Leica Microsystems $\mathrm{GmbH}$ ).

Electron microscopy. Cardiac ventricles were processed for electron microscopy analysis as described (19). Mouse myocardium was perfused with $2.5 \%$ glutaraldehyde at for $24 \mathrm{~h}$ at $25^{\circ} \mathrm{C}$ (Tianjin Kemiou Chemical Reagent Co., Ltd.; 25\% glutaraldehyde: $4 \% \mathrm{PFA}=1: 9)$. The harvested tissues were post-fixed in $2.5 \%$ glutaraldehyde for $24 \mathrm{~h}$ at $25^{\circ} \mathrm{C}$, subsequently stained in $1 \%$ osmium tetroxide for $72 \mathrm{~h}$ and then cleared in propylene oxide post-dehydration in a series at $25^{\circ} \mathrm{C}$ for 7 days, For transmission electron microscopy (TEM; HT7800; Hitachi, Ltd.), $50 \mathrm{~nm}$ ultrathin sections were acquired and double-stained with $2 \%$ uranyl acetate and lead citrate for TEM analysis.
Statistical analysis. Statistical analyses were performed using GraphPad Prism 5 software (GraphPad Software, Inc.). All experiments were repeated at least three times and data are presented as the mean \pm SD. Comparisons between two groups were analyzed using the unpaired Student's test. Comparison of measurement data between two groups was analyzed by t-test, while comparison of measurement data among multiple groups was analyzed by one-way (Tukey) analysis of variance. $\mathrm{P}<0.05$ was considered to indicate a statistically significant difference.

\section{Results}

shRNA-mediated PINCH1 knockdown attenuates cell viability and migration, and increases cell apoptosis. To explore the significance of PINCH1 in the function of HL1 cells, cell viability, migration and apoptosis were assessed in HL1 cells transfected with PINCH1 shRNA. Furthermore, rescue experiments were performed by inducing PINCH1 overexpression (PINCH1 OE) in shRNA PINCH1-transfected HL1 cells, as previously described (20). Successful shRNA PINCH1 transfection was confirmed by western blotting (Fig. 1A). Following PINCH1 OE, the RT-qPCR and western blotting results demonstrated that PINCH1 expression was higher in the PINCH1 OE group compared with that in the NC OE group (Fig. S1). Moreover, the transfected cells expressing exogenous GFP were enriched using flow cytometry (Fig. 1B). PINCH1 OE also significantly upregulated PINCH1 expression in shRNA PINCH1-transfected HL1 cells $(\mathrm{P}<0.05$; Fig. 1C). Additionally, compared with control and shRNA PINCH1 + PINCH1 OE groups, cell viability and migration were significantly decreased in the shRNA PINCH1 group $(\mathrm{P}<0.01)$ (Fig. 1D and E). Subsequently, the effect of PINCH1 on HL1 cell apoptosis was significantly evaluated by flow cytometry. The results showed that the apoptotic rate of HL1 cells transfected with shRNA PINCH1 was significantly increased compared with control and shRNA PINCH1 + PINCH1 OE groups $(\mathrm{P}<0.01$; Fig. $1 \mathrm{~F})$.

HL1 cell apoptosis is associated with the $N F-\kappa B$ signaling pathway. To determine whether shRNA PINCH1-mediated HL1 cardiomyocyte apoptosis was associated with the NF- $\kappa \mathrm{B}$ signaling pathway, western blotting was performed to evaluate the expression levels of NF- $\kappa \mathrm{B}$-related proteins in shRNA NC and shRNA PINCH1-transfected HL1 cells. Compared with those in the WT group, the protein expression levels of $\mathrm{NF}-\kappa \mathrm{B}$, MYD88, TNF- $\alpha$ and caspase-3 were significantly increased, whereas the expression levels of MMP-2 and MMP-9 were significantly decreased in shRNA PINCH1-transfected HL1 cells $(\mathrm{P}<0.05$; Fig. $2 \mathrm{~A}$ and $\mathrm{B})$. These data suggested that the biological effect of shRNA PINCH1-transfected HL1 cells might be mediated by the NF- $\mathrm{B}$ signaling pathway.

PINCH1 KO may lead to exacerbated cardiac injury. To further evaluate the potential effects of PINCH1 in the myocardium, a mouse model with PINCH1 KO in cardiac cells was established. Subsequently, MI was induced in PINCH1 KO and WT mice at 18 weeks of age. The western blotting results demonstrated the successful KO of PINCH1 in the myocardial tissue (Fig. 3A). In addition, all mice were 

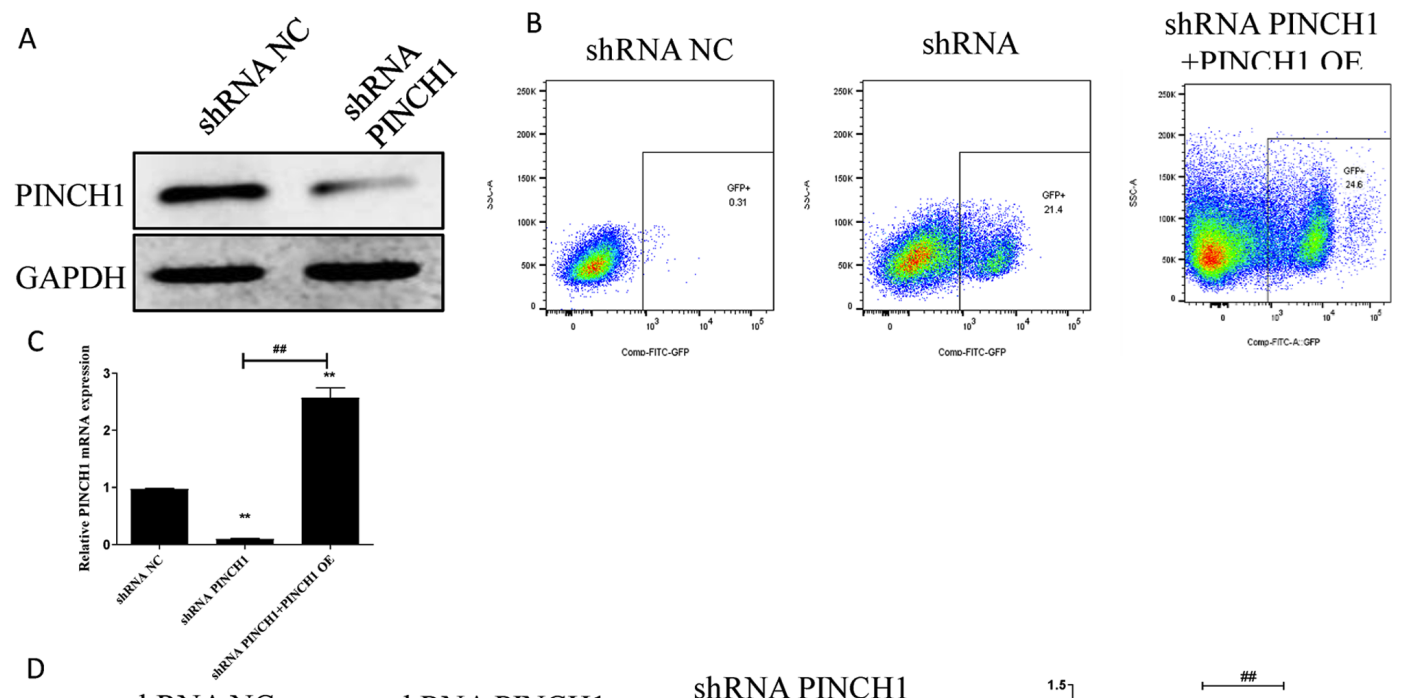

ShRNA NC

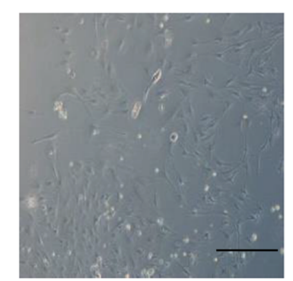

$E$

ShRNA NC

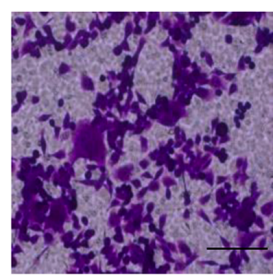

$\mathrm{F}$

shRNA NC

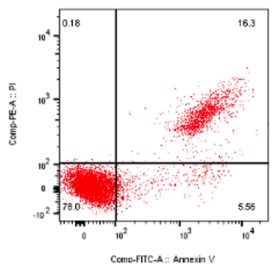

shRNA PINCH1

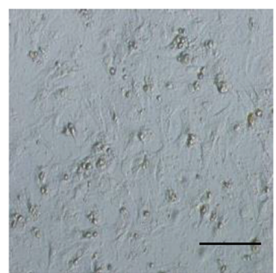

shRNA

PINCH1

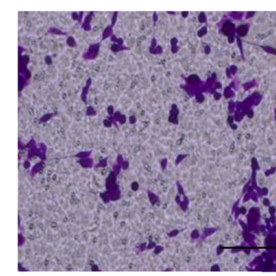

shRNA

$\mathrm{PINCH} 1$

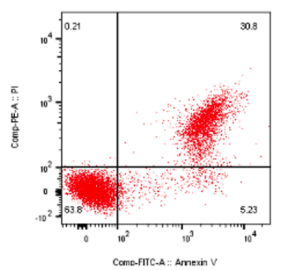

shRNA PINCH1

$+\mathrm{PINCH1} \mathrm{OE}$

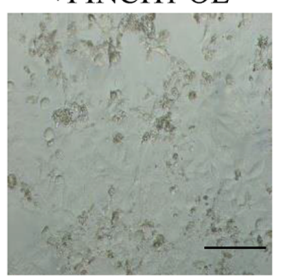

ShRNA PINCH1

$+\mathrm{PINCH} 1 \mathrm{OE}$

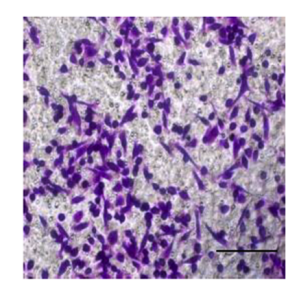

ShRNA PINCH1

+ PINCH1 OE

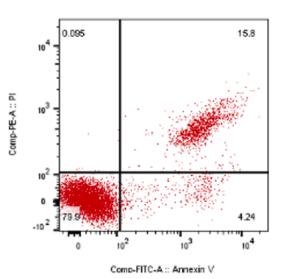

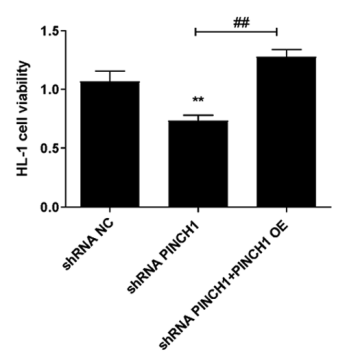
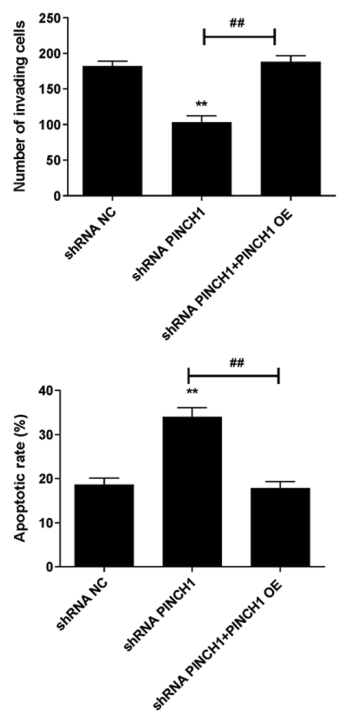

Figure 1. Effect of PINCH1 on the function of HL1 cells. (A) Successful PINCH1 shRNA was confirmed by western blot analysis. (B) Percentage of GFP ${ }^{+}$cells in PINCH1 shRNA/PINCH1 OE cells following transfection. Untreated cells were used as a negative control. (C) The expression levels of PINCH1 mRNA in control, PINCH1 shRNA, and PINCH1 shRNA/PINCH1 OE cells were determined by qPCR. (D) HL1 cell viability (magnification, $x 100)$ and (E) migration ability were decreased following PINCH1 shRNA (magnification, x100). (F) HL1 cell apoptosis rate was significantly increased following PINCH1 shRNA. ${ }^{* *} \mathrm{P}<0.05$ vs. shRNA NC; ${ }^{\# \#} \mathrm{P}<0.05$ vs. PINCH1 SHRNA/PINCH1 OE group. PINCH1, particularly interesting new cysteine histidine rich 1; PINCH1 shRNA, PINCH1 knockdown; PINCH1 OE, PINCH1 overexpression; PINCH1 SHRNA/PINCH1 OE, PINCH1 overexpression (PINCH1 OE) in PINCH1 shRNA HL1 cells.

examined for acute MI by electrocardiogram via ultrasound (Fig. 3B). All PINCH1 KO mice gradually developed severe growth retardation with postnatal development from one weeks to eight weeks old (Fig. 3C). The H\&E staining results showed obvious swelling and hypertrophy of myocardial cells in the myocardial tissue cross sectional area of PINCH1 KO mice Histological examination showed myocardial cell arrangement disorders of left ventricle compared with control hearts (Fig. 3D). Furthermore, the Masson's trichrome staining results showed that myocardial fibers in the PINCH1 KO group were disordered and displayed obvious fibrotic changes compared with those in the WT group (Fig. 3E). The TUNEL staining assay results demonstrated that the apoptotic rate of cardiomyocytes in the PINCH1 KO group was significantly increased compared with that in the WT group (Fig. 3F). The aforementioned findings indicated that PINCH1 KO caused myocardial fibrosis and heart failure in mice. 
A
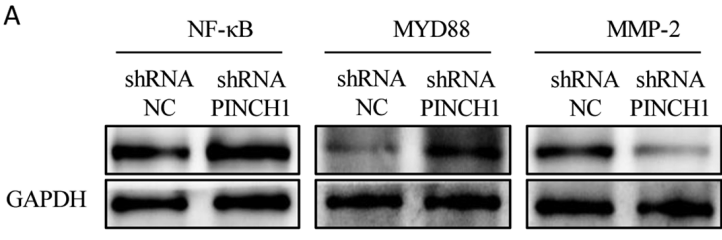

GAPDH
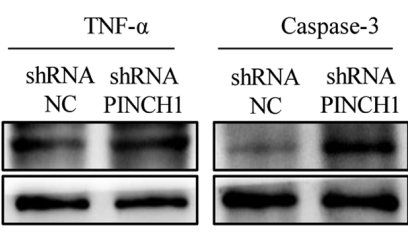

B

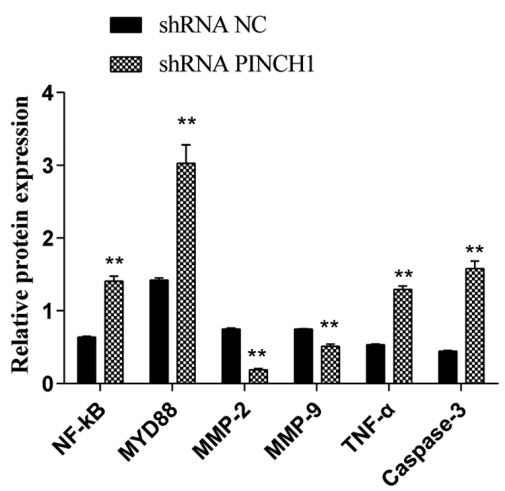

Figure 2. HL1 cell apoptosis is associated with the NF- $\mathrm{kB}$ signaling pathway. Expression levels of NF- $\mathrm{B}$ signaling pathway-related proteins were (A) determined by western blotting and (B) semi-quantified. NF- $\mathrm{KB}$, MYD88, TNF- $\alpha$ and caspase-3 expression levels were significantly increased, whereas MMP-2 and MMP-9 expression levels were notably decreased in the shRNA PINCH1 group. ${ }^{* *} \mathrm{P}<0.01 \mathrm{vs.} \mathrm{shRNA} \mathrm{NC}$ group. MYD88, myeloid differentiation factor 88; PINCH1, particularly interesting new cysteine histidine rich 1; WT, wild-type; shRNA, short hairpin RNA.

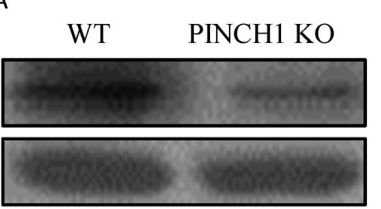

C

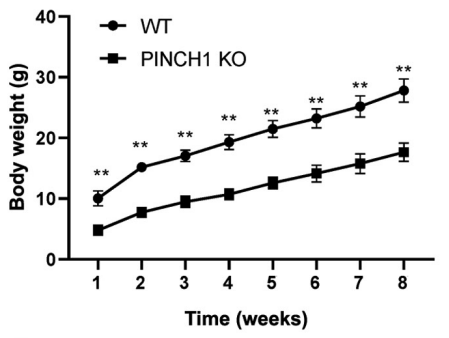

WT

PINCH1 KO

D

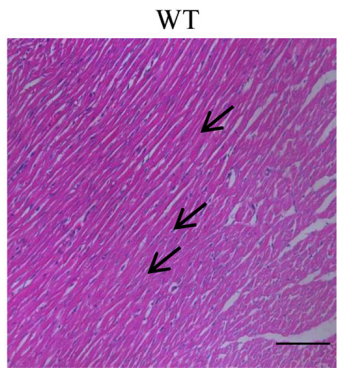

MI

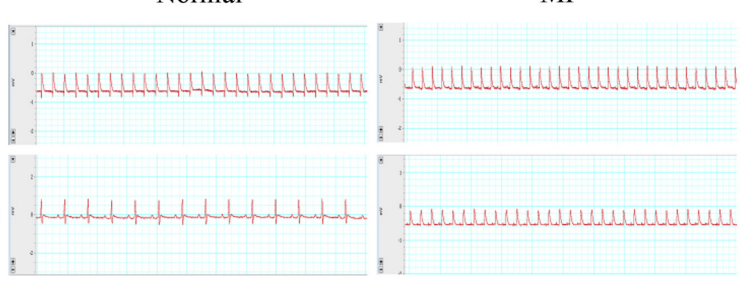

PINCH1 KO

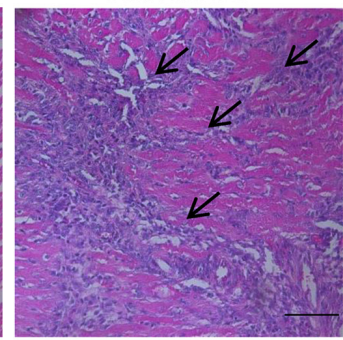

WT

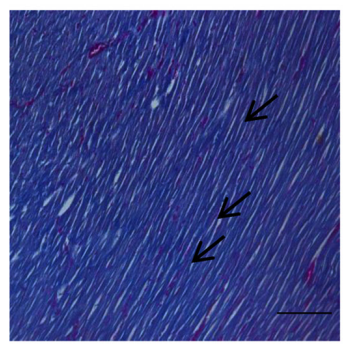

$\mathrm{F}$
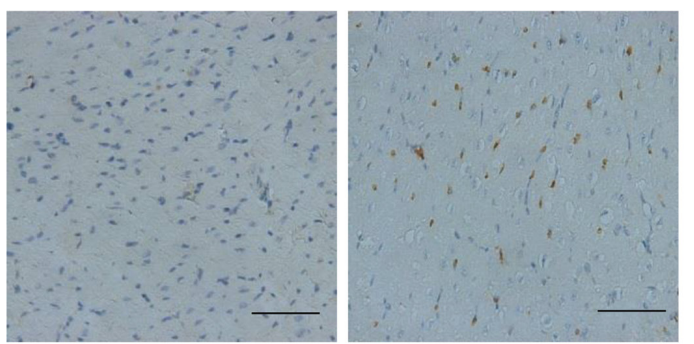

PINCH1 KO

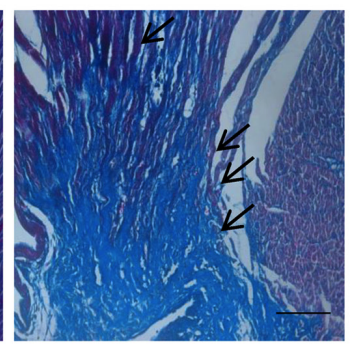

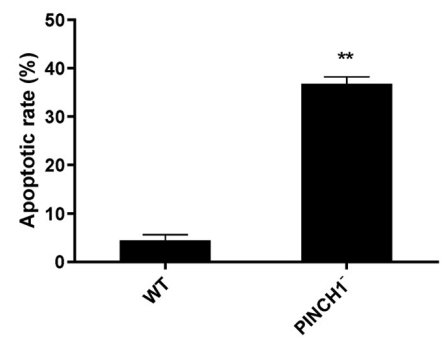

Figure 3. Myocardial PINCH1 KO may lead to exacerbated cardiac injury. (A) Efficient PINCH1 KO in the myocardium as demonstrated by western blotting. (B) Electrocardiograms were performed in each mouse by ultrasound. (C) PINCH1 KO mouse growth was gradually slowed down from one to eight weeks old. (D) H\&E (magnification, x200) and (E) Masson's trichrome (magnification, x200) staining demonstrated notably increased myocardial injury in the PINCH1 KO group. (F) Cell apoptosis in different groups was determined by performing TUNEL assays (magnification, $\mathrm{x} 200$ ). ${ }^{* *} \mathrm{P}<0.05$ vs. WT. PINCH1, particularly interesting new cysteine histidine rich $1 ; \mathrm{KO}$, knockout; $\mathrm{H} \& \mathrm{E}$, hematoxylin and eosin. 


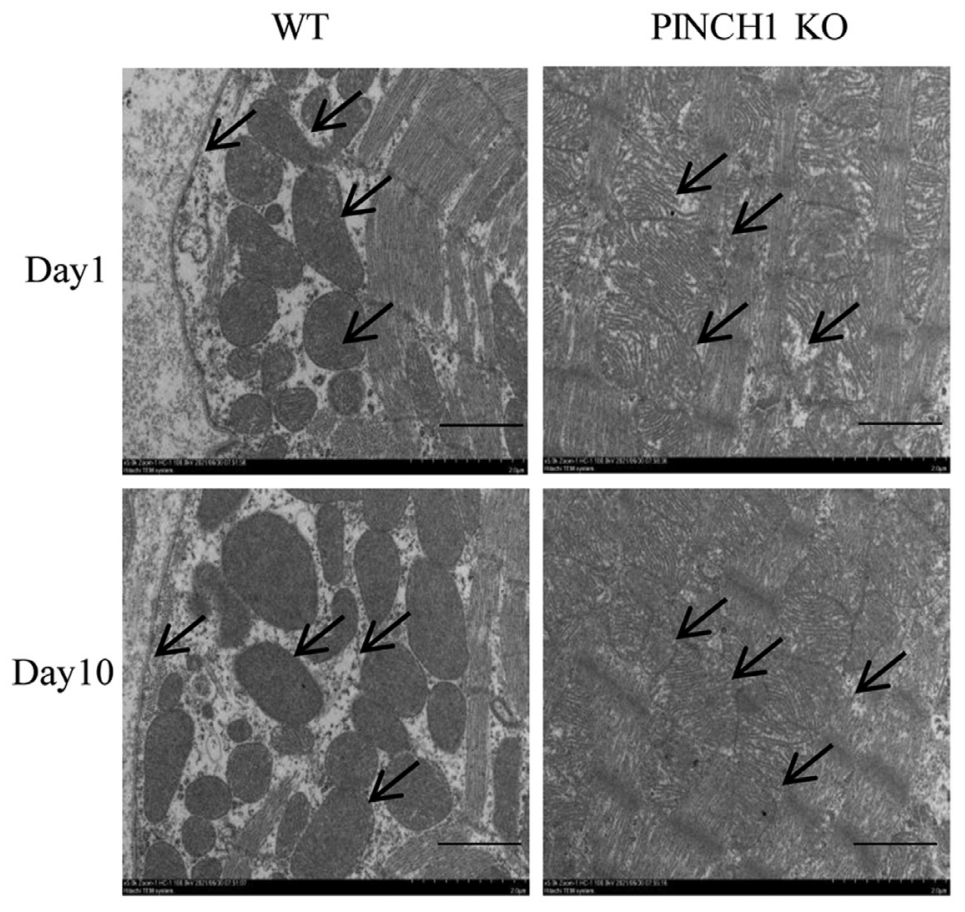

Figure 4. Representative electron micrographs of PINCH1 KO mice. At day 1 after birth, intercalated disk structures remained largely similar between control and PINCH1 KO. However, in a few focal regions, gaps of intercalated disks were widened, with disarrayed sarcomeric structure and distorted Z lines. At day 10 after birth, intercalated disks of control samples were clearly observed, connecting adjacent myocytes end to end. In PINCH1 KO, membranes of the intercalated disk were highly convoluted, and gaps of the intercalated disks were significantly widened (scale bar=2 $\mu \mathrm{m}$ ). PINCH1, particularly interesting new cysteine histidine rich 1; KO, knockout; WT, wild-type.
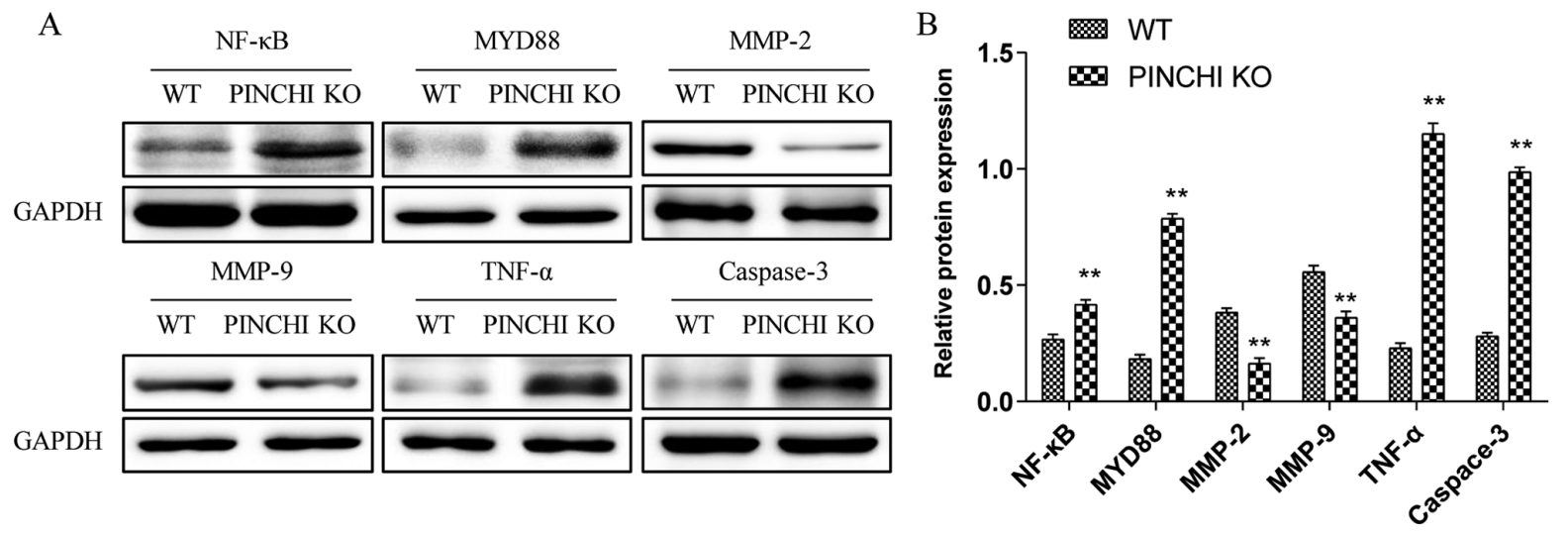

Figure 5. Myocardial PINCH1 KO induces cardiomyopathy in mice via the NF- $\mathrm{KB}$ signaling pathway. Expression levels of NF- $\mathrm{B}$ signaling pathway-related proteins were (A) determined by western blotting and (B) semi-quantified. NF-кB, MYD88, TNF- $\alpha$ and caspase-3 expression levels were significantly upregulated, whereas MMP-2 and MMP-9 expression levels were downregulated in the myocardial tissue of PINCH1 KO mice. "** $<0.01$ vs. shRNA NC group. PINCH1, particularly interesting new cysteine histidine rich 1; KO, knockout; WT, wild-type; MYD88, myeloid differentiation factor 88.

Heart ultrastructural abnormalities in PINCH1 KO mice. In representative electron micrographs at day 1 , intercalated disk structures remained largely similar between control and PINCH1 KO. However, in a few focal regions, gaps of intercalated disks were widened, with disarrayed sarcomeric structure and distorted $\mathrm{Z}$ lines. At day 10, intercalated disks of control samples were clearly observed, connecting adjacent myocytes end to end. In PINCH1 KO, membranes of the intercalated disk were highly convoluted, and gaps of the intercalated disks were significantly widened. Compared with WT mice, the muscle layer structure was disorganized, some myofibrils were broken, the distance between the membrane and the muscle layer of myocytes was obviously widened, and the decreased adhesion ability of the adjacent intercellular myocytes resulted in an increased cell gap in the PINCH1 KO group (Fig. 4).

PINCHI KO in the myocardium may promote cardiomyopathy in mice via the $N F-\kappa B$ signaling. The results of the in vitro experiments demonstrated that $\mathrm{PINCH} 1 \mathrm{KO}$-induced HL1 cardiomyocyte apoptosis was associated with the NF- $\kappa B$ signaling pathway. Therefore, to investigate whether the PINCH1 KO-induced cardiomyopathy in mice was also mediated by NF- $\kappa \mathrm{B}$ signaling, the expression levels of the $\mathrm{NF}-\kappa \mathrm{B}$ pathway-related proteins were determined in cardiac 
tissues by western blotting. Consistent with the in vitro results, compared with the WT group, the expression levels of $\mathrm{NF}-\kappa \mathrm{B}$, MYD88, TNF- $\alpha$ and caspase-3 were significantly increased $(\mathrm{P}<0.01$; Fig. 5), whereas the expression levels of MMP-2 and MMP-9 were notably decreased in the myocardial tissues of the PINCH1 KO group ( $\mathrm{P}<0.01$; Fig. 5).

\section{Discussion}

Cardiovascular diseases are a major health problem worldwide. As the leading cause of death among patients with cardiovascular disease, MI exhibits high morbidity and mortality rates worldwide. Furthermore, MI is a very common ischemic heart disease characterized by impaired cardiomyocyte function, resulting in progressive heart failure (21-25). PINCH1 serves a crucial role in maintaining the structure and function of the heart and is involved in several biological processes, including cell migration and survival. In zebrafish, PINCH1 knockdown led to severe heart failure via the ILK-parvin-PINCH/protein kinase B signaling pathway (26). In addition, PINCH1 KO in mouse embryos resulted in attenuated ventricular cardiomyocyte proliferation and excessive cell death (27). The present study demonstrated that shRNA-mediated PINCH1 knockdown in HL1 cardiomyocytes also decreased cell viability and migration, but increased apoptosis. Additionally, targeted PINCH1 KO in the myocardium of mice aggravated cardiomyopathy and heart failure.

MI has been associated with inflammatory responses in the myocardium. Although, the myocardial tissue inflammatory response promotes myocardial repair and wound healing, excessive inflammation may also lead to heart failure. Following MI, surviving myocardial and inflammatory cells promote NF- $\kappa \mathrm{B}$ activation to participate in the inflammatory repair response and initiate the inflammatory cascade. However, the release of excessive inflammatory cytokines, MMPs and other inflammatory-associated factors also expands the MI area $(24,25)$. TNF- $\alpha$ is a proinflammatory cytokine involved in the regulation of numerous autoimmune diseases. The downstream target of TNF- $\alpha$-mediated inflammation is the transcription factor $\mathrm{NF}-\kappa \mathrm{B}$. Therefore, $\mathrm{NF}-\kappa \mathrm{B}$ activation in the heart tissue is considered as a hallmark of MI (10). It has been reported that toll-like receptors promote heart failure in response to injury or stress by activating the $\mathrm{NF}-\kappa \mathrm{B}$ signaling pathway via the adapter protein MYD88. MYD88 serves an important role in the signaling and activation of $\mathrm{NF}-\kappa \mathrm{B}$ downstream signals (18). Furthermore, MMPs are proteolytic inflammation-related enzymes involved in the degradation and remodeling of extracellular matrix. It has been reported that MMPs promote the ventricular remodeling process (28-31). The results from the in vivo and in vitro experiments conducted in the present study revealed that the expression levels of NF- $\mathrm{B}, \mathrm{MYD} 88$, TNF- $\alpha$, and caspase-3 were significantly increased, whereas MMP-2 and MMP-9 expression levels were decreased in the shRNA PINCH1 and PINCH1 KO groups.

In summary, the present study demonstrated that targeted PINCH1 shRNA attenuated cell viability and migration, and increased cell apoptosis in vitro, thus leading to myocardial disease and heart failure in mice via the $N F-\kappa B$ signaling pathway. These findings provided a theoretical basis for the clinical treatment of MI. However, since two homologous PINCH isomers have been identified, the mechanism of action underlying $\mathrm{PINCH}$ in $\mathrm{MI}$ requires further investigation.

\section{Acknowledgements}

Not applicable.

\section{Funding}

The present study was supported by the Key Discipline Groups of Shanghai Pudong New Area (grant no. PWZxq2017-01), the Natural Science Foundation of Shanghai of China (grant no. 17ZR1425800) and Shanghai Municipal Health Commission of China (grant no. 202040159).

\section{Availability of data and materials}

The datasets used and/or analyzed during the current study are available from the corresponding author on reasonable request.

\section{Authors' contributions}

YZ conceived and designed the experiments. XW and JS contributed to designing the study and drafted the manuscript. $\mathrm{XW}$ and $\mathrm{YZ}$ confirm the authenticity of all the raw data. XW, JS, ZL and KL performed the data analysis. YZ made substantial contributions to proofreading the manuscript and gave final approval of the version to be published. All authors read and approved the final manuscript.

\section{Ethics approval and consent to participate}

The animal experiments involved in the present study were approved by the Ethics Committee of Zhoupu Hospital (approval no. 2017-C-040-E01).

\section{Patient consent for publication}

Not applicable.

\section{Competing interests}

The authors declare that they have no competing interests.

\section{References}

1. Sopko N, Qin Y, Finan A, Dadabayev A, Chigurupati S, Qin J, Penn MS and Gupta S: Significance of thymosin $\beta 4$ and implication of PINCH-1-ILK- $\alpha$-parvin (PIP) complex in human dilated cardiomyopathy. PLoS One 6: e20184, 2011.

2. Zou L, Ma X, Lin S, Wu B, Chen Y and Peng C: Bone marrow mesenchymal stem cell-derived exosomes protect against myocardial infarction by promoting autophagy. Exp Ther Med 18: 2574-2582, 2019.

3. Zhang HR, Bai H, Yang E, Zhong ZH, Chen WY, Xiao Y, $\mathrm{Gu}$ YH and Lu SF: Effect of moxibustion preconditioning on autophagy-related proteins in rats with myocardial ischemia reperfusion injury. Ann Transl Med 7: 559, 2019.

4. Wang R, Wang M, Zhou J, Ye T, Xie X, Ni D, Ye J, Han Q, Di C, Guo L, et al: Shuxuening injection protects against myocardial ischemia-reperfusion injury through reducing oxidative stress, inflammation and thrombosis. Ann Transl Med 7: 562, 2019. 
5. Jiang T, Zhang L, Ding M and Li M: Protective effect of vasicine against myocardial infarction in rats via modulation of oxidative stress, inflammation, and the PI3K/Akt pathway. Drug Des Devel Ther 13: 3773-3784, 2019.

6. Zhang C, Liang R, Gan X, Yang X, Chen L and Jian J: MicroRNA-384-5p/Beclin-1 as potential indicators for epigallocatechin gallate against cardiomyocytes ischemia reperfusion injury by inhibiting autophagy via PI3K/Akt pathway. Drug Des Devel Ther 13: 3607-3623, 2019.

7. Belete S, Punjabi K, Afoke J and Anderson J: Surgical management of post-infarction ventricular septal defect, mitral regurgitation and ventricular aneurysm. J Surg Case Rep 2019: rjz256, 2019.

8. Liang X, Sun Y, Ye M, Scimia MC, Cheng H, Martin J, Wang G, Rearden $\mathrm{A}, \mathrm{Wu} \mathrm{C}$, Peterson KL, et al: Targeted ablation of PINCH1 and PINCH2 from murine myocardium results in dilated cardiomyopathy and early postnatal lethality. Circulation 120 568-576, 2009.

9. Braun A, Bordoy R, Stanchi F, Moser M, Kostka GG, Ehler E, Brandau $\mathrm{O}$ and Fässler R: PINCH2 is a new five LIM domain protein, homologous to PINCH and localized to focal adhesions. Exp Cell Res 284: 239-250, 2003.

10. Liang X, Zhou Q, Li X, Sun Y, Lu M, Dalton N, Ross J Jr and Chen J: PINCH1 plays an essential role in early murine embryonic development but is dispensable in ventricular cardiomyocytes. Mol Cell Biol 25: 3056-3062, 2005.

11. Pan SP, Pirker T, Kunert O, Kretschmer N, Hummelbrunner S, Latkolik SL, Rappai J, Dirsch VM, Bochkov V and Bauer R: C13 megastigmane derivatives from epipremnum pinnatum: $\beta$-Damascenone inhibits the expression of pro-inflammatory cytokines and leukocyte adhesion molecules as well as NF- $\mathrm{BB}$ signaling. Front Pharmacol 10: 1351, 2019.

12. Zhang J, Lei JR, Yuan LL, Wen R and Yang J: Response gene to complement-32 promotes cell survival via the NF- $\kappa B$ pathway in non-small-cell lung cancer. Exp Ther Med 19: 107-114, 2020.

13. Ge ZW, Wang BC, Hu JL, Sun JJ, Wang S, Chen XJ, Meng SP, Liu L and Cheng ZY: IRAK3 gene silencing prevents cardiac rupture and ventricular remodeling through negative regulation of the NF- $\kappa \mathrm{B}$ signaling pathway in a mouse model of acute myocardial infarction. J Cell Physiol 234: 11722-11733, 2019.

14. Han A, Lu Y, Zheng Q, Zhang J, Zhao Y, Zhao M and Cui X Qiliqiangxin attenuates cardiac remodeling via inhibition of TGF- $\beta 1 / \mathrm{Smad} 3$ and NF- $\kappa \mathrm{B}$ signaling pathways in a rat model of myocardial infarction. Cell Physiol Biochem 45: 1797-1806, 2018

15. He Q, Zhou W, Xiong C, Tan G and Chen M: Lycopene attenuates inflammation and apoptosis in post-myocardial infarction remodeling by inhibiting the nuclear factor- $\kappa \mathrm{B}$ signaling pathway. Mol Med Rep 11: 374-378, 2015.

16. Jin JL, Deng ZT, Lyu RG, Liu XH and Wei JR: Expression changes of Notch and nuclear factor- $\mathrm{kB}$ signaling pathways in the rat heart with myocardial infarction. Zhonghua Xin Xue Guan Bing Za Zhi 45: 507-512, 2017 (In Chinese).

17. Gupta S, Kumar S, Sopko N, Qin Y, Wei C and Kim IK: Thymosin $\beta 4$ and cardiac protection: Implication in inflammation and fibrosis. Ann N Y Acad Sci 1269: 84-91, 2012.

18. Qiu P, Wheater MK, Qiu Y and Sosne G: Thymosin beta4 inhibits TNF-alpha-induced NF-kappaB activation, IL-8 expression, and the sensitizing effects by its partners PINCH-1 and ILK. FASEB J 25: 1815-1826, 2011

19. Chen J, Kubalak SW, Minamisawa S, Price RL, Becker KD, Hickey R, Ross J Jr and Chien KR: Selective requirement of myosin light chain $2 \mathrm{v}$ in embryonic heart function. J Biol Chem 273: 1252-1256, 1998
20. Sandfort V, Eke I and Cordes N: The role of the focal adhesion protein PINCH1 for the radiosensitivity of adhesion and suspension cell cultures. PLoS One 5: e13056, 2010.

21. Zhang W, Tian Y, Gao Q, Li X, Li Y, Zhang J, Yao C, Wang Y, Wang $\mathrm{H}$, Zhao $\mathrm{Y}$, et al: Inhibition of apoptosis reduces diploidization of haploid mouse embryonic stem cells during differentiation. Stem Cell Reports 15: 185-197, 2020.

22. Yokokawa T, Yoshihisa A, Kanno Y, Abe S, Misaka T, Yamada S, Kaneshiro T, Sato T, Oikawa M, Kobayashi A, et al: Circulating acetoacetate is associated with poor prognosis in heart failure patients. Int J Cardiol Heart Vasc 25: 100432, 2019.

23. Luo ZR, Li H, Xiao ZX, Shao SJ, Zhao TT, Zhao Y, Mou FF, Yu B and Guo HD: Taohong siwu decoction exerts a beneficial effect on cardiac function by possibly improving the microenvironment and decreasing mitochondrial fission after myocardial infarction. Cardiol Res Pract 2019: 5198278, 2019.

24. Neyrinck K, Breuls N, Holvoet B, Oosterlinck W, Wolfs E, Vanbilloen H, Gheysens O, Duelen R, Gsell W, Lambrichts I, et al: The human somatostatin receptor type 2 as an imaging and suicide reporter gene for pluripotent stem cell-derived therapy of myocardial infarction. Theranostics 8: 2799-2813, 2018.

25. Puddighinu G, D'Amario D, Foglio E, Manchi M, Siracusano A, Pontemezzo E, Cordella M, Facchiano F, Pellegrini L, Mangoni A, et al: Molecular mechanisms of cardioprotective effects mediated by transplanted cardiac $\mathrm{ckit}^{+}$cells through the activation of an inflammatory hypoxia-dependent reparative response. Oncotarget 9: 937-957, 2017.

26. Wang B, Zhang L, Cao H, Yang J, Wu M, Ma Y, Fan H, Zhan Z and Liu Z: Myoblast transplantation improves cardiac function after myocardial infarction through attenuating inflammatory responses. Oncotarget 8: 68780-68794, 2017.

27. Meder B, Huttner IG, Sedaghat-Hamedani F, Just S, Dahme T, Frese KS, Vogel B, Köhler D, Kloos W, Rudloff J, et al: PINCH proteins regulate cardiac contractility by modulating integrin-linked kinase-protein kinase B signaling. Mol Cell Biol 31: 3424-3435, 2011.

28. Singh MV, Swaminathan PD, Luczak ED, Kutschke W, Weiss RM and Anderson ME: MyD88 mediated inflammatory signaling leads to CaMKII oxidation, cardiac hypertrophy and death after myocardial infarction. J Mol Cell Cardiol 52: 1135-1144, 2012.

29. Gu C, Wang F, Zhao Z, Wang H, Cong X and Chen X: Lysophosphatidic acid is associated with atherosclerotic plaque instability by regulating NF- $\kappa \mathrm{B}$ dependent matrix metalloproteinase-9 expression via $\mathrm{LPA}_{2}$ in macrophages. Front Physiol 8: 266, 2017.

30. Wang Q, Sui X, Sui DJ and Yang P: Flavonoid extract from propolis inhibits cardiac fibrosis triggered by myocardial infarction through upregulation of SIRT1. Evid Based Complement Alternat Med 2018: 4957573, 2018

31. Ibarra-LaraL,Sánchez-AguilarM,Soria-CastroE,Vargas-BarrónJ, Roldán FJ, Pavón N, Torres-Narváez JC, Cervantes-Pérez LG, Pastelín-Hernández G and Sánchez-Mendoza A: Clofibrate treatment decreases inflammation and reverses myocardial infarction-induced remodelation in a rodent experimental model. Molecules 24: 270, 2019.

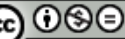

This work is licensed under a Creative Commons Attribution-NonCommercial-NoDerivatives 4.0 International (CC BY-NC-ND 4.0) License. 